\title{
Phonon-Mediated Anomalous Dynamics of Defects
}

\author{
Ali Najafi and Ramin Golestanian \\ Institute for Advanced Studies in Basic Sciences, Zanjan 45195-159, Iran
}

(November 13, 2018)

\begin{abstract}
Dynamics of an array of line defects interacting with a background elastic medium is studied in the linear regime. It is shown that the inertial coupling between the defects and the ambient phonons leads to an anomalous response behavior for the deformation modes of a defect-lattice, in the form of anisotropic and anomalous mass and elastic constants, resonant dissipation through excitation of phonons, and instabilities. The case of a single fluctuating line defect is also studied, and it is shown that it could lead to formation of shock waves in the elastic medium for sufficiently high frequency deformation modes.
\end{abstract}

Topological defects have a wide range of interest in many branches of physics. Vortices in superconductors, superfluids, and Bose-Einstein condensates, crystal defects in solid bodies, topological excitations in spin systems with continuous symmetry, and defects in liquid crystals are famous examples of their ubiquity [1]. While they play a pivotal role in our fundamental understanding of the superfluid [2] and melting [3] transitions in two dimensions, they are also important in determining the properties of real materials - for example they are responsible to a large degree for the plastic behavior of solid bodies [4].

Over the past decade, the problem of defect dynamics has attracted a considerable attention mainly in describing the dynamics of defect-mediated phase transitions [5], and in determining the physical properties of vortex-lattices in type-II supercondoctors [6, 8, 9]. However, the interaction of defects with the excitations of the elastic background, or phonons, is not taken into account in these studies, because in the context of equilibrium statistical thermodynamics the defects are completely separated from the phonons and there is no net coupling between the two degrees of freedom [10]. Quantized phonons, however, are shown to interact with defects leading to interesting effects [11 13]. The interesting observation of a collection of quantized vortices in Bose-Einstein condensates has also raised new questions about the dynamical behavior of defects 14].

Here, we consider the classical dynamics of a collection of line defects in the form of a regular array, which are dynamically fluctuating in an ambient elastic medium away from thermodynamic equilibrium. The inertial coupling between the defects and the phonons of the background medium is shown to lead to a plethora of novel effects in the long time- and length-scale collective dynamics of the defect-lattice: (i) The deformation modes of the defect-lattice acquire an anomalous transverse mass, which diverges for modes that are in resonance with the background phonons, and a finite longitudinal mass. (ii) There is a corresponding anomalous transverse elastic constant in the direction parallel to the line defects, as well as a corresponding finite longitudinal elastic con- stant. (iii) An effective anomalous friction appears at the resonance, signalling the transfer of mechanical energy from the defect-lattice to the elastic phonons. (iv) The defect-lattice becomes intrinsically unstable for frequencies higher than the phononic resonance frequency for each wavevector, due to the enhanced excitation of phonons. (v) The elastic moduli in the perpendicular direction are anomalous, and show an intrinsic instability for the shear modes. The case of a single fluctuating line defect is also studied. It is shown that deformation modes along the line defect with a phase velocity higher than the bulk velocity of phonons lead to the creation of shock waves. The propagation of phonons in the periodic matrix of the defects is also studied, and it is proposed that such arrangements may lead to formation of frequency gaps in the phononic band structure.

We consider a simple scalar elasticity described by the field $\theta(\mathbf{r}, t)$, which could be a component of the displacement field in crystals, or the phase of the order parameter for superfluids. The field is then conveniently decomposed into two parts as $\theta(\mathbf{r}, t)=\theta_{\mathrm{ph}}(\mathbf{r}, t)+\theta_{\text {def }}(\mathbf{r}, t)$ : (i) a singular part $\theta_{\text {def }}(\mathbf{r}, t)$ that is a solution of the defect condition of the form $\oint d \mathbf{l} \cdot \nabla \theta=2 \pi n$, where the integral is taken over any closed path around the defect and $n$ is the corresponding winding number or the topological charge of the defect, and (ii) a nonsingular part $\theta_{\mathrm{ph}}(\mathbf{r}, t)$ that describes the phononic degrees of freedom in the elastic medium. While such a representation assumes a vanishing size for the defects, we should keep in mind that in reality they always maintain a finite core size, which is of the order of the atomic lattice constant $a$ in crystals. With the above decomposition, we then consider an action as

$$
\mathcal{A}=\int d t d^{3} \mathbf{r}\left[\frac{\rho}{2}\left(\partial_{t} \theta_{\mathrm{ph}}+\partial_{t} \theta_{\mathrm{def}}\right)^{2}-\frac{J}{2}\left(\nabla \theta_{\mathrm{ph}}+\nabla \theta_{\mathrm{def}}\right)^{2}\right]
$$

where the coefficient $\rho$ is a (linear) mass density, and $J$ is a stiffness coefficient. The quantity $c=\sqrt{J / \rho}$ is the phase velocity of sound waves in the medium. 


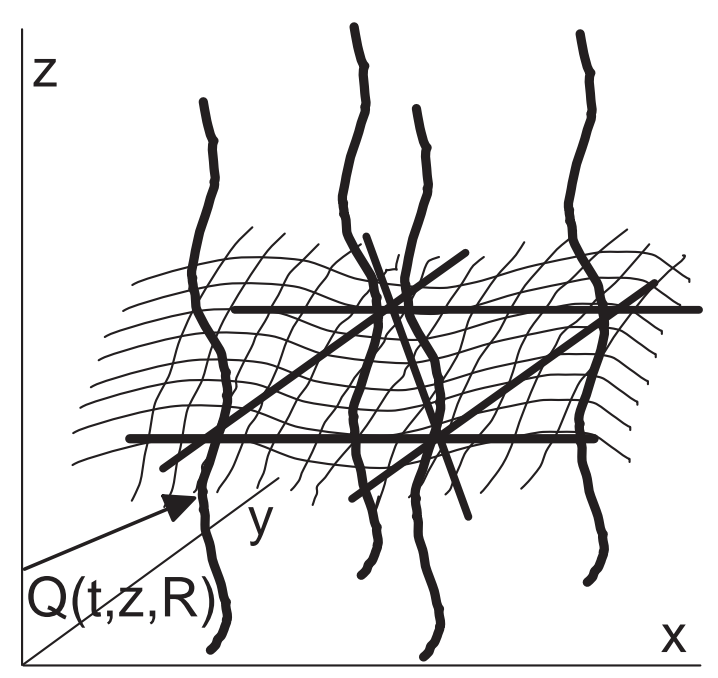

FIG. 1. Array of fluctuating line defects in an elastic medium. The deformation modes of the defect lattice are coupled to the phonons of the background medium.

To study the many-body effects in the dynamics of line defects mediated by phonons, we consider a system consisting of many line defects (with $n=1$ ) that are arranged on a triangular lattice (when at equilibrium) as shown in Fig. 1. In this case the defect field is given as

$$
\theta_{\text {def }}\left(\mathbf{r}_{\perp},\{\mathbf{Q}(\mathbf{R}, z, t)\}\right)=\sum_{\{\mathbf{R}\}} \arctan \frac{y-Q_{2}(\mathbf{R}, z, t)}{x-Q_{1}(\mathbf{R}, z, t)}
$$

where $\mathbf{r}_{\perp}=(x, y)$, and $\mathbf{Q}(\mathbf{R}, z, t)=$ $\left(Q_{1}(\mathbf{R}, z, t), Q_{2}(\mathbf{R}, z, t)\right)$ is the two dimensional position vector of the core of a line defect in the $x$ - $y$ plane, which is fluctuating around a point $\mathbf{R}$ of a regular two dimensional lattice (see Fig. 1). The corresponding Euler-Lagrange equation for the phonon field then reads

$$
\left[\partial_{t}^{2}-c^{2} \nabla^{2}\right] \theta_{\mathrm{ph}}(\mathbf{r}, t)=-\left[\partial_{t}^{2}-c^{2} \partial_{z}^{2}\right] \theta_{\mathrm{def}}\left(\mathbf{r}_{\perp},\{\mathbf{Q}(\mathbf{R}, z, t)\}\right) .
$$

Note that $\nabla_{\perp}^{2} \theta_{\text {def }}=0$. The above equation implies that the system of fluctuating defects acts as a complicated source for phononic waves - each defect can transmit or absorb phonons from the medium during its motion. We will see below that the net result of this coupling is a dissipative effect in which the moving defects lose their energy in the form of phonon radiation.

To study the collective dynamics of the defects in the presence of phonons, we should eliminate the phononic degrees of freedom in the action, and obtain an effective action for the defect degrees of freedom $\{\mathbf{Q}(\mathbf{R}, z, t)\}$. For this purpose, we assume that the line defects are only slightly distorted from their equilibrium configurations, so that their positions can be described as $\mathbf{Q}(\mathbf{R}, z, t)=$ $\mathbf{R}+\mathbf{u}(\mathbf{R}, z, t)$ with $\mathbf{u}(\mathbf{R}, z, t)$ being much less than the defect lattice constant $b$. We then solve the wave equation for the phonons [Eq. (3)] perturbatively in the deformation field $\mathbf{u}(\mathbf{R}, z, t)$, and obtain the effective action up to the leading order as

$$
\begin{aligned}
\mathcal{A}_{\text {eff }}= & \frac{1}{2} \int_{\mathbf{q} \in \text { B.z. }} \frac{d^{2} \mathbf{q}}{(2 \pi)^{2}} \frac{d k_{z}}{2 \pi} \frac{d \omega}{2 \pi} \chi_{\alpha \beta}\left(\mathbf{q}, k_{z}, \omega\right) \\
& \times u_{\alpha}\left(\mathbf{q}, k_{z}, \omega\right) u_{\beta}\left(-\mathbf{q},-k_{z},-\omega\right),
\end{aligned}
$$

where

$$
\begin{aligned}
\chi_{\alpha \beta}\left(\mathbf{q}, k_{z}, \omega\right) & =J \sum_{\mathbf{G}}\left\{\left[\frac{G_{\alpha} G_{\beta}}{G^{2}}-\frac{\left(q_{\alpha}+G_{\alpha}\right)\left(q_{\beta}+G_{\beta}\right)}{(\mathbf{q}+\mathbf{G})^{2}}\right]\right. \\
& \left.+\frac{\left(\omega^{2}-c^{2} k_{z}^{2}\right)\left[\frac{\left(q_{\alpha}+G_{\alpha}\right)\left(q_{\beta}+G_{\beta}\right)}{(\mathbf{q}+\mathbf{G})^{2}}-\delta_{\alpha \beta}\right]}{\omega^{2}-c^{2} k_{z}^{2}-c^{2}(\mathbf{q}+\mathbf{G})^{2}}\right\} .
\end{aligned}
$$

and $\{\mathbf{G}$ \} represents the reciprocal lattice of the original defect-lattice $\{\mathbf{R}\}$. Note that the kernel consists of two contributions: (i) a term which does not depend on frequency and comes from the usual long-range logarithmic interaction between the line defects, and (ii) a term with dynamical origin describing an additional interaction between the defects mediated by phonons.

The summation over all the reciprocal lattice vectors of the two dimensional lattice in the above kernel is difficult to perform exactly. However, we can study the long time- and length-scale elasticity of the defect lattice by focusing on the small frequency and wavevector limit in Eq. (5). This can be achieved by separating the $\mathbf{G}=0$ term and expanding the other terms in powers of $q / G$, $k_{z} / G$, and $\omega /(c G)$. In this case the summation over $\mathbf{G}$ can be performed and we obtain an analytical expression for the kernel to the leading order as

$$
\begin{aligned}
\chi_{\alpha \beta}\left(\mathbf{q}, k_{z}, \omega\right) & =\left(M_{T} \omega^{2}-K_{T}^{\|} k_{z}^{2}-K_{T}^{\perp} q^{2}\right)\left(\delta_{\alpha \beta}-\hat{q}_{\alpha} \hat{q}_{\beta}\right) \\
& +\left(M_{L} \omega^{2}-K_{L}^{\|} k_{z}^{2}-K_{L}^{\perp} q^{2}\right) \hat{q}_{\alpha} \hat{q}_{\beta} \\
& +i \omega \zeta_{T}\left(\delta_{\alpha \beta}-\hat{q}_{\alpha} \hat{q}_{\beta}\right),
\end{aligned}
$$

where the effective transverse and longitudinal mass densities are given as

$$
M_{T}\left(q, k_{z}, \omega\right)=\frac{\rho}{q^{2}+k_{z}^{2}-(\omega / c)^{2}}+\frac{\rho}{\kappa^{2}}, \quad M_{L}=\frac{\rho}{\kappa^{2}},
$$

the effective transverse and longitudinal elastic moduli in the parallel direction as

$$
K_{T}^{\|}\left(q, k_{z}, \omega\right)=\frac{J}{q^{2}+k_{z}^{2}-(\omega / c)^{2}}+\frac{J}{\kappa^{2}}, \quad K_{L}^{\|}=\frac{J}{\kappa^{2}},
$$

the effective transverse and longitudinal elastic moduli in the perpendicular direction as

$$
K_{T}^{\perp}(q)=-\frac{J}{2 q^{2}}, \quad K_{L}^{\perp}(q)=\frac{J}{2 q^{2}},
$$

and, finally, the effective transverse friction coefficient as

$$
\zeta_{T}\left(q, k_{z}, \omega\right)=\frac{\pi J c^{2} q^{2}}{|\omega|} \delta\left(\omega^{2}-c^{2} k_{z}^{2}-c^{2} q^{2}\right) .
$$


In the above, parallel and perpendicular are defined with respect to the line defects, and the screening length $\kappa^{-1}$ is defined as

$$
\kappa^{-2}=\frac{1}{2} \sum_{\mathbf{G} \neq 0} \frac{1}{G^{2}} \simeq \frac{\pi \ln (b / a)}{G^{* 2}},
$$

where $G^{*}$ is the lattice constant of the reciprocal lattice, which for a triangular lattice of line defects is given as $G^{*}=\frac{4 \pi}{\sqrt{3} b}$. Note that the real (imaginary) part of the above kernel is even (odd) in $\omega$ and this ensures that the response is causal.

The above results show that the dynamics of a defect lattice in an elastic medium is anomalous. While the longitudinal deformation modes acquire finite mass and elastic modulus in the parallel direction due to coupling with phonons, the corresponding mass and elastic modulus in the parallel direction for the transverse modes are frequency and wavevector dependent, and diverge in the small wavevector and frequency limit. Moreover, whereas the transverse mass and elastic modulus in the parallel direction are positive for $\omega^{2}<c^{2}\left(q^{2}+k_{z}^{2}\right)$, they diverge when the frequency and wavevectors of the deformation modes hit that of a possible phononic excitation in the background. In this case the defect-lattice dynamics becomes dissipative - as manifested by the appearance of an imaginary part in the response kernel that defines an effective friction coefficient - which corresponds to transfer of mechanical energy from the defect lattice to the phonons. For $\omega^{2}>c^{2}\left(q^{2}+k_{z}^{2}\right)$, the transverse mass and elastic modulus in the parallel direction become negative, signalling an instability in the defect-lattice due to the resonant coupling with the background phonons. The transverse and longitudinal elastic moduli in the perpendicular direction are also wavevector dependent, and they reveal an inherent instability in the deformation modes that is related to the well-known instability of a system of charges in electrostatics; often alluded to as the Earnshaw's theorem [9, 15].

It is also interesting to consider the case where all the line defects are frozen (to straight lines) and only a single defect is fluctuating, which can be achieved by assuming that $u_{\alpha}\left(\mathbf{q}, k_{z}, \omega\right)$ does not depend on $\mathbf{q}$. We can then combine the restricted $\mathbf{q}$ integration in the first Brillouin zone and the summation over the reciprocal lattice vectors $\mathbf{G}$ to a free integration over $\mathbf{q}$, and calculate the response kernel $\chi_{\mathrm{sl}}\left(k_{z}, \omega\right) \delta_{\alpha \beta}=\int_{\text {B.Z. }} \frac{d^{2} \mathbf{q}}{(2 \pi)^{2}} \chi_{\alpha \beta}\left(\mathbf{q}, k_{z}, \omega\right)$. This yields

$$
\chi_{\mathrm{sl}}\left(k_{z}, \omega\right)=\rho_{\mathrm{sl}} \omega^{2}-K_{\mathrm{sl}} k_{z}^{2}+i \omega \zeta_{\mathrm{sl}},
$$

with the effective linear mass density for a single line defect given as

$$
\rho_{\mathrm{sl}}\left(k_{z}, \omega\right)=\frac{\pi}{2} \rho \ln \left(\frac{\omega_{\mathrm{D}}^{2}}{\left|\omega^{2}-c^{2} k_{z}^{2}\right|}\right),
$$

the effective elastic modulus as

$$
K_{\mathrm{sl}}\left(k_{z}, \omega\right)=\frac{\pi}{2} J \ln \left(\frac{\omega_{\mathrm{D}}^{2}}{\left|\omega^{2}-c^{2} k_{z}^{2}\right|}\right),
$$

and the effective friction coefficient as

$$
\zeta_{\mathrm{sl}}\left(k_{z}, \omega\right)=\frac{\pi^{2}}{2} \rho \frac{\left(\omega^{2}-c^{2} k_{z}^{2}\right)}{|\omega|} \Theta\left(\omega^{2}-c^{2} k_{z}^{2}\right) .
$$

In the above, the Debye frequency $\omega_{\mathrm{D}} \simeq c / a$ is a high frequency cutoff in the system, and $\Theta$ represents the Heaviside step function.

The effective mass density and elastic modulus for the line defect are logarithmically larger than the nominal mass density and stiffness of the material in the core of the vortex, and they are also frequency and wavevector dependent. While they are positive for all frequency and wavevectors, they logarithmically diverge when $\omega=c k_{z}$, and in particular in the limit $\omega \rightarrow 0$ and $k_{z} \rightarrow 0$.

In the case where the phase velocity of waves on the line defect $\omega / k_{z}$ is greater than the phonon velocity in the system $c$, the response kernel in Eq. 12) develops an imaginary part, which is a reflection of a transfer of mechanical energy from the fluctuating defect to the elastic medium in the form of radiating phonons. This effect is similar to the Cherenkov radiation, where a charged particle moving in a dielectric medium with superluminal velocity (i.e. a velocity greater than the velocity of photons in that medium) radiates electromagnetic waves.

The time-averaged energy dissipation rate for the fluctuating line defect can be calculated as

$$
P=\int \frac{d k_{z}}{2 \pi} \frac{d \omega}{2 \pi} \zeta_{\mathrm{sl}}\left(k_{z}, \omega\right) \omega^{2}\left|\mathbf{u}\left(k_{z}, \omega\right)\right|^{2} .
$$

To explicitly check that the dissipation is due to phonon radiation, we calculate the rate of change in energy for the phonon field as

$$
\frac{d E_{\mathrm{ph}}}{d t}=\int d^{3} \mathbf{r} \frac{\partial}{\partial t} T_{00}
$$

using the 00-component of the stress tensor $T_{\mu \nu}$ [16] that can be calculated from the phonon field action in Eq. (1). The result comes out exactly equal to the dissipation rate as given in Eq. (16) above.

To estimate the radiated power, we consider an example in which the defect is undergoing a solid harmonic motion described via $\mathbf{u}\left(k_{z}, \omega\right)=\mathbf{u}_{0}(2 \pi)^{2} \delta\left(k_{z}\right)\left[\delta\left(\omega+\omega_{0}\right)+\right.$ $\left.\delta\left(\omega-\omega_{0}\right)\right] / 2$. The time-averaged radiated power per unit length from this oscillating line defect is then calculated from Eq. (16) as $P / L=\pi^{2} \rho u_{0}^{2} \omega_{0}^{3} / 4$.

Finally, we note that the periodic arrangement of the defect lines on a lattice changes the spectrum of phonons, as in the case of electrons in metals. This is manifested in the singular points (poles) in the dynamical term in the response kernel of Eq. (5) whose structure determines the dispersion relation for the phononic excitations. The dispersion relation $\Omega(\mathbf{q}) \equiv \sqrt{\omega(q)^{2}-c^{2} k_{z}^{2}}=c|\mathbf{q}-\mathbf{G}|$ should respect the symmetry of the reciprocal lattice $\mathbf{G}$. 
As an example, the phononic dispersion diagram for the case of a triangular lattice of line defects is plotted in Fig. 2. The unit vectors for the triangular lattice are $b(1,0)$ and $b\left(\frac{1}{2}, \frac{\sqrt{3}}{2}\right)$, and the unit vectors for the corresponding reciprocal lattice are $G^{*}\left(\frac{\sqrt{3}}{2},-\frac{1}{2}\right)$ and $G^{*}(0,1)$, where $G^{*}=\frac{4 \pi}{\sqrt{3} b}$ is the lattice constant of the reciprocal lattice.

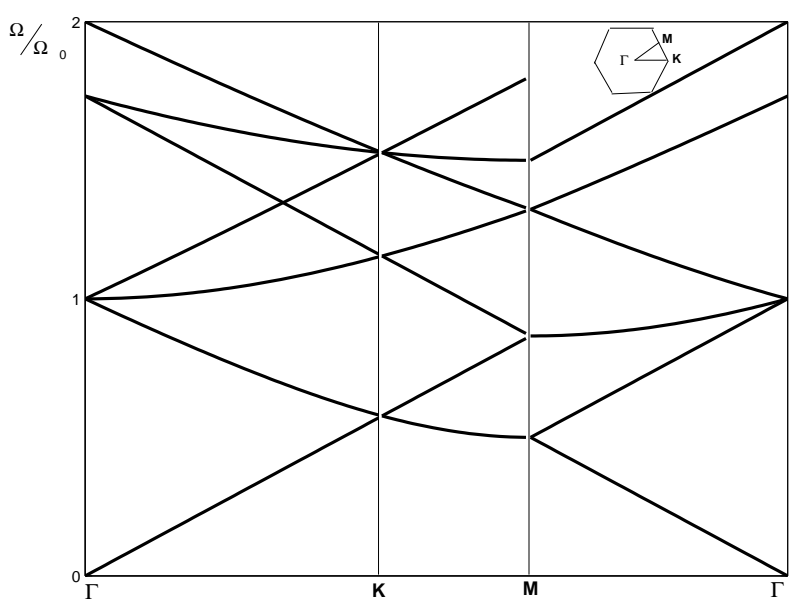

FIG. 2. Diagram of phononic dispersion relation defined via $\Omega(\mathbf{q}) \equiv \sqrt{\omega(q)^{2}-c^{2} k_{z}^{2}}=c|\mathbf{q}-\mathbf{G}|$ in a triangular lattice of line defects, with $\Omega_{0}=c G^{*}=\frac{4 \pi c}{\sqrt{3} b}$. The inset shows the first Brillouin zone of the triangular lattice.

While the band diagram in Fig. 2 has no gaps, one can argue that an interaction of the phonons with the interior of the defects that changes the propagation velocity of phonons from $c$ to $c / \sqrt{\epsilon(\mathbf{r})}$ in the vicinity of the defects [7] can produce a gap in the band diagram, similar to the case of photonic crystals [17]. A simple calculation (similar to the estimation of the gap for electrons in a weak periodic potential 仼) then shows that such an interaction can lead to frequency gaps in the zone boundary of the order of $\Delta \sim c G^{*}(a / b)$, where $a$ is the core size of the defect [18]. This may suggest that a rather dense array of defects may act as a "phononic crystal," which could be of potential interest for applications.

In conclusion, we have shown that the dynamical interaction of defects with phonons can lead to renormalization of mass and dissipation, which is analogous to the radiation-reaction force for electrons and their mass renormalization due to their interaction with electromagnetic fields [19].

We are grateful to J. Sethna for very helpful comments, and to S. Ramanathan for invaluable discussions during the early stages of this work.

[1] P.M. Chaikin and T.C. Lubensky, Principles Of Condensed Matter physics(Cambridge University Press, 1997).

[2] J.M. Kosterlitz and D.J. Thouless, J. Phys. C 6, 1181 (1973).

[3] D.R. Nelson and B.I. Halperin, Phys. Rev. B 19, 2457 (1979).

[4] N. Ashcroft and D. Mermin, Solid State Physics, (Holt, Rinehart, Winston, 1976).

[5] C. Domb, J.L. Lebowitz, Phase Transition and Critical Phenomena, Vol. 7 (Academic Press, 1983).

[6] G. Blatter, M.V. Feigel'man, V.B. Geshkenbein, A.I. Larkin and V.M. Vinokour, Rev. Mod. Phys. 66, 1125 (1994).

[7] P. Minnhagen, Rev. Mod. Phys. 54, 1001 (1987).

[8] C.J. Olson, C. Reichhardt, and F. Nori, Phys. Rev. Lett. 81, 3757 (1998).

[9] M.-C. Miguel and M. Kardar, Phys. Rev. B 62, 5942 (2000).

[10] J.V. Jose, L.P. Kadanoff, S. Kirkpatrick, and D.R. Nelson, Phys. Rev. B 16, 1217 (1977).

[11] J.P. Sethna, Phys. Rev. B 24, 698 (1981); Phys. Rev. B 25, 5050 (1982).

[12] T. Vegge, J.P. Sethna, S.-A. Cheong, K.W. Jacobsen, C.R. Myers, and D.C. Ralph, Phys. Rev. Lett. 86, 1546 (2001).

[13] D. J. Thouless, P. Ao, and Q. Niu, Phys. Rev. Lett. 76, 3758 (1996); C. Wexler and D.J. Thouless, Phys. Rev. B 58, R8897 (1998).

[14] M.R. Matthews, B.P. Anderson, P.C. Haljan, D.S. Hall, C.E. Wieman, and E.A. Cornell, Phys. Rev. Lett. 83 2498 (1999); K.W. Madison, F. Chevy, W. Wohlleben, and J. Dalibard, Phys. Rev. Lett. 84806 (2000); S. Inouye, S. Gupta, T. Rosenband, A.P. Chikkatur, A. Grlitz, T.L. Gustavson, A.E. Leanhardt, D.E. Pritchard, and W. Ketterle, Phys. Rev. Lett. 87, 080402 (2001).

[15] R.P. Feynman, R. Leighton, M. Sands, The Feynman Lectures on Physics, Vol. 2 (Addison-Wesley, 1975).

[16] L.D. Landau and E.M. Lifshitz, The Classical Theory of Fields 4th edition (Pergamon, Oxford, England, 1975).

[17] J.D. Joannoupoulos, R.B. Meade, and J.N. Winn, Photonic Crystals, (Princeton University Press, 1995).

[18] A. Najafi and R. Golestanian, to be published.

[19] A.O. Barut, Electrodynamics and Classical Theory of Fields and Particles. (Dover Publications, New York, 1980). 